\title{
Cancer Compass: Examining Personal Health Record Usage Among Breast Cancer Survivors
}

\author{
Maia Jacobs, James Clawson and Elizabeth Mynatt \\ School of Interactive Computing \\ College of Computing \\ Georgia Institute of Technology \\ Atlanta, GA \\ mjacobs30@gatech.edu
}

\begin{abstract}
As personal health records (PHRs) become more common, they have the potential to become a critical tool for breast cancer survivors. Our research has identified a hierarchy of needs that could be addressed, in part, through PHR capabilities. While breast cancer survivors are expected to receive many benefits from using PHRs, little is known about how people will use and adopt this technology. In order to gain a better understanding of the potential uses and pitfalls for PHRs, we will give 80 breast cancer survivors mobile PHR systems and monitor their usage over the course of two months. We will investigate the motivational factors that lead to PHR adoption and the correlations between system usage, self-efficacy and locus of control. Our goal is to understand if PHRs have the potential to successfully engage breast cancer survivors with their daily health practices.
\end{abstract}

Keywords-cancer compass; breast cancer; personal health records

\section{INTRODUCTION}

With recent technology advances, it will soon become common practice for people to easily access and manage their health information on a daily basis. The hope is that the technologies that support these functions will engage users and empower people to take control of their healthcare, leading to better health behaviors and health outcomes.

Engagement may be particularly important for improving the healthcare experience for breast cancer survivors (where survivorship includes all people living with breast cancer from the time of diagnosis), as this disease constitutes the largest population of cancer survivors [3], with a population of over 2.6 million in the United States [10]. Survivors often struggle with obtaining basic information regarding their health status and finding sufficient time to interact with their oncologists [11]. Current cancer care systems often fail to provide survivors with the ability to easily access and process personal health information [5].

Electronic personal health records (PHRs) have the potential to become a crucial tool to educate breast cancer survivors about their condition and engage them with their health. Although the definition of a PHR is continuously developing, we consider a PHR to be a tool that provides users with a medical summary, tracking capabilities, and a method for communicating with one's provider [9]. While this

Sponsored by: Georgia Department of Community Health technology is beginning to be deployed on a larger scale, cancer survivors are also showing an interest in using PHRs to organize personal heath data [2]. This combination of technology readiness and interest may allow for significant changes in cancer care and survivor engagement.

Research is beginning to show the benefits that PHRs can have in supporting patients, especially with information management [7]. However, many questions remain in the area of PHR adoption. In order to determine the success of current PHR designs, we must better understand how and why PHR systems engage users. We seek to examine this question through the Cancer Compass project, a study of a PHR system deployed to breast cancer survivors.

\section{RESEARCH QUESTIONS}

We have two research questions guiding the Cancer Compass project. Our first question centers on motivation, and which specific factors motivate survivors to adopt high usage patterns of PHRs. We expect there will be numerous factors that influence PHR adoption, including the stage of cancer, severity of treatment, and amount of time an individual spends interacting directly with their health providers. We hope that busy schedules and complex disease management act as encouragement, rather than deterrence, for using this technology. Further, we hope that survivors who face common struggles such as finding information about their disease or communicating with their provider are motivated to accomplish these tasks with the help provided by the PHRs.

We also intend to investigate whether PHR usage correlates to changes in self-efficacy and locus of control, which have been deemed significant measures for health behavior change [1]. We would anticipate that survivors who are able to use PHRs to complete important tasks, such as recording and sharing health information, would display an increase in selfefficacy in managing healthcare through technology. Further, if survivors are able to successfully initiate communication with their providers through this technology we would expect to see a shift towards internal locus of control. We believe both of these questions will help us understand whether current PHR technologies will be effective in engaging breast cancer survivors to take a more central role in their healthcare. 


\section{COMPLETED WORK}

In order to deploy a useful PHR system, we first sought to gain a better understanding of the healthcare system currently experienced by breast cancer survivors and the perceived needs in the target community. Over a 4-month period in 2012, we conducted 17 interviews and 6 focus groups. We met with 30 members of a rural community, including 7 breast cancer survivors, 5 caregivers (family members or friends), 8 cancer navigators (professionals who help cancer survivors navigate the complex healthcare system by providing support and informational resources), and 10 health care professionals. We used semi-structured interviews focusing on current technology use, important points of communication in the medical treatment process, and the overall survivorship experience from the perspective of these key players.

Going into this project, we assumed that the majority of needs to be addressed by the PHR would be informational in nature, such as information about cancer treatments, treatment options, coordination of appointments, interpretation of lab results, etc. However, our informants discussed a much larger breadth of needs and priorities. Through these interviews we developed a hierarchical list of essential needs (see Figure 1), which include basic needs such as transportation, food, and benefits. Additional needs include emotional and spiritual wellbeing and increased health information/knowledge. Through the interviews we uncovered that access to resources was the motivating factor that drove user needs. This observation means that if a survivor already possessed access to the necessary health benefits and could easily travel to all healthcare appointments, the focus shifted to the need for emotional support or health information. The hierarchy of needs is significant to our future Cancer Compass deployment. For PHR technology to possess the potential to engage survivors in their healthcare, survivors must be able to use the tool to support their individual needs. We will use the findings from this work to ensure that the technology will support the anticipated needs of all breast cancer survivors in the community regardless of where they are on the hierarchy.

\section{CANCER COMPASS SYSTEM}

Through the Cancer Compass project we aim to study how a real-world deployment of PHRs affects breast cancer survivors. Microsoft HealthVault will act as the backend data store for this project and we will deploy our PHR technologies on Google Nexus tablets. Tablets were chosen for this project as past research has shown that many important tasks, such as scheduling appointments, often occur outside of the home [7]. Mobile devices will be better able to support these types of tasks. Key features of the Cancer Compass tablet include the user's medical summary, a symptom tracker, and a secure information exchange system that allows survivors to email with their providers. We will also add applications from the eHealth Initiative's Cancer Resource Guide [4] in order to provide a robust system that can be used to address the full list of survivors' needs identified in our previous work.

We will deploy Cancer Compass PHR systems to 80 breast cancer survivors in a rural community and evaluate their utilization over a 2-month period. This large scale deployment

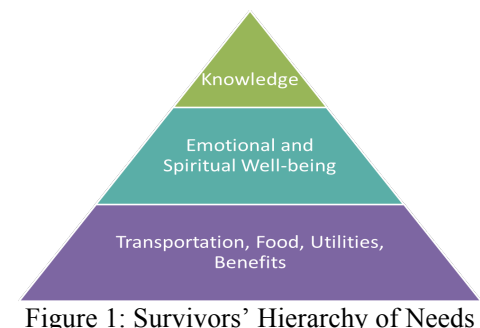

will allow us to monitor near-term adoption and usage patterns. During this time, we will collect and analyze log files regularly. We wish to observe which functions of the system are used and when. For example, we will distinguish between survivors using the PHR to (a) respond to messages from their healthcare providers, (b) log symptoms at home (c) transmit questions or unsolicited data and (d) manage resources and information. We will be coupling log file data with interviews, surveys, and journaling to provide additional insight into the initial motivations of the survivors, their perceptions of the PHR usefulness and usability and their overall usage patterns.

We predict that this work can help determine the potential impact that PHRs may have on engaging breast cancer survivors in their own healthcare. We also anticipate that this research will help identify areas of improvement for future PHR designs. Some potential challenges with PHR systems have already been defined [8]. We expect to learn whether such barriers affect usage among breast cancer survivors and provide suggestions to improve PHR adoption and enhance the breast cancer survivorship experience.

\section{REFERENCES}

[1] Bandura, A., "Health promotion by social cognitive means," Health Education \& Behavior, vol. 31, pp. 143-164 (2004).

[2] Beckjord, E.B., Rechis, R., Hesse, B.W., "Use of, preferences for, and expectations regarding personal health records among people affected by cancer: results of a LIVESTRONG survey and the 2008 Health Information National Trends Survey," Proceedings of the $45^{\text {th }}$ International Conference on System Sciences, Hawaii, (2012).

[3] Cappiello, M., Cunningham, R.S., Tish Knobf, M., Erdos, D., "Breast cancer survivors: information and support after treatment," Clinical Nursing Research, vol. 16, pp. 278-293, (2007).

[4] eHealth Initiative.(2013). Health IT Cancer Resource Guide. [online]. http://www.ehealthinitiative.org/health-it-cancer-resource.html

[5] Hayes, G., Abowd, G.D., Davis, J.S., Blount, M.L., Ebling M., Mynatt, E.D., "Opportunities for pervasive computing in chronic cancer care," Proceedings of the 6th International Conference on Pervasive Computing, Sydney, Australia, (2008).

[6] http://www.wolframalpha.com/input/? $i=$ floyd + county $+g a+$ income

[7] Klasnja, P., Hartzler, A., Powell, C., Pratt, W., "Supporting cancer patients' unanchored health information management with mobile technology," AMIA Annual Symposium Proceedings, (2011).

[8] Liu, L.S., Shih, P.C., Hayes, G.R., "Barriers to the Adoption and Use of Personal Health Record Systems," iConference, Seattle, WA, (2011).

[9] Stead, W.W., "Rethinking electronic health records to better achieve quality \& safety goals," Annual Review of Medicine, (2006).

[10] U.S. Breast Cancer Statisitics (2012), [online]. http://www.breastcancer.org/symptoms/understand_bc/statistics

[11] Unruh, K.T., Pratt, W. "The invisible work of being a patient and implications for health care: '[the doctor is] my business partner in the most important business in my life, staying alive," Ethnographic Praxis in Industry Conference Proceedings, pp. 40-50, (2008). 